\title{
The discrepancy between mean reward and mean reinforcement'
}

\author{
CHARLES C. PERKINS, JR., JEAN C. LEVIS, STEPHEN SULZBACHER AND DAVID F. BERGER
}

EMORY UNIVERSITY

\begin{abstract}
Two groups of 12 rats were run in a single choice maze where one arm contained one unit of food $100 \%$ of the time, the other two units $50 \%$ of the time. For Group S one unit was two $45 \mathrm{mg}$ pellets; for Group L one unit was 12 pellets. Group $\mathrm{L}$ acquired a significant preference for the 1 unit-100\% side, and Group S acquired a significant preference for the 2 units $-50 \%$ side.

\section{Problem}

Leventhal, Morgan, Morrell, \& Perkins (1959) reported that in a single choice maze, rats came to select the turn followed by $.25 \mathrm{gm}$ half the time and no food the other half. However, they found no significant preference for either response when one was followed by $1 \mathrm{gm}$ of food on every occasion and the other by 2 gm on half the trials and nothing on the other half. Similary, with small water reward (1 unit equals two drops) they obtained a preference for 2 units $-50 \%$ of the time over 1 unit-100\%, but when one unit equalled 10 drops, no preference was acquired. On the other hand, unpublished work in the Emory Laboratory by R. G. Seymann using a two-bar Skinner box failed to provide evidence of a preference for 2 units-50\% over 1 unit-100\% with small units of food reward. The present study was designed to replicate part of the experiment by Leventhal et al (1959) under slightly different conditions.
\end{abstract}

\section{Method}

A single choice point $\mathrm{E}$ maze was employed. The starting box and alley before the choice point were grey; the left hand side was black and the right hand side white. The goal boxes were not visable from the choice point. Guillotine doors were located at the exit of the starting box, at each side of the choice point, and at the entrance to the goal boxes.

The Ss were 24 albino rats from the Emory colony, maintained at $80 \%$ of free feeding weight by feedings shortly after the daily experimental periods. For two successive days before the beginning of training, Ss were placed in the maze in groups of four or six with all doors open for approximately $15 \mathrm{~min}$. Also on these days Ss were given .045 gm Noyes pellets in food dishes in their home cage to familiarize them with the reward to be employed. On each day of training $S$ received free trials until he had gone to one side for half his day's runs after which he was forced to the opposite side for the remainder of the day's runs.

All S's received two units of reward on half of each day's runs to one side in an unpredictable sequence and one unit of reward on all runs to the other side. On each of the first two days $S$ received four trials; eight trials were given on each of the next 29 days. One unit of reward was two Noyes pellets for Group S and 12 Noyes pellets for Group L. Group L was run in squads of four Ss; Group S in squads of six. The intertrial interval was approximately $15 \mathrm{~min}$. for both groups. One squad in each group was run by each of two Es (J.C.L. and S.S.); another E (D.F.B.) ran the third squad in Group L (which did not differ systematically from the others). Within each squad the 1 unit- $100 \%$ side was on the right for half the Ss and other counterbalancing was like that of Leventhal et al (1959). E's were not aware of the results obtained by Leventhal et al (1959). Two days of running were skipped for one squad in Group $L$, one between days 24 and 25, the other between days 26 and 27. The deprivation schedule was maintained, and no effect of missing a day was apparent.

\section{Results}

The results are presented in Fig. 1 which shows that the small reward Ss acquired a marked preference for the 2 unit-50\% side, and the large reward Ss acquired a marked preference for the 1 unit-100\% side. On the first half of the day's runs for the last 10 days combined, 11 of $12 \mathrm{Ss}$ in each group ran to the same side on over half the trials. This preference is significant on the binomial distribution $(p<.003)$.

The results for Group $S$ are quite similar to those of Leventhal et al (1959) for their small reward group (one unit $=.25 \mathrm{gm}$ Purina Lab Chow). Results of Group $\mathrm{L}$ are quite different from those reported by Leventhal

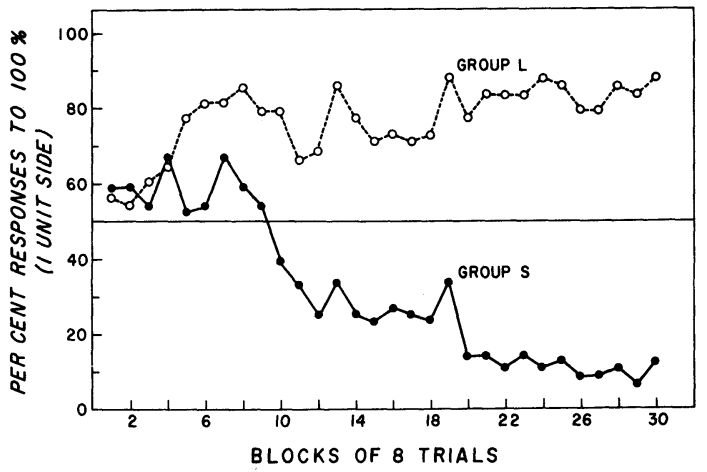

Fig. 1. Learning curves for the two groups. Percentages of the first half of the days runs which were to the 1 unit $100 \%$ side are plotted for each block of eight trials (including all runs) for each group. 
et al (1959) for any of their groups even the one for which one unit was $1.0 \mathrm{gm}$ of Lab Chow. There is no obvious explanation for this difference. However, there were differences in apparatus, reward magnitude, type of reward, and strain of Ss.

It is of interest that the preference was acquired faster by Group L than by Group S. The difference between the two groups in rate of acquisition of a preference is just what one would expect from the suggestion of Leventhal et al that the preference of small reward groups is dependent on the acquisition of more appropriate concomitant responses with the larger (i.e., 2 unit) reward. These concomitant responses would have to have been conditioned before the preference would begin to show up. In the case of Group L, the preference for the 1 unit-100\% condition may be dependent on a decrease in the reinforcing effect of food when hunger drive is decreased, i.e., while the lasthalf of a two unit reward is consumed. This effect can be expected to operate from the very first trial.

\section{Reference}

Leventhal, A. M., Morrell, R. E., Morgan, E. F., \& Perkins, C. C. The relation between mean reward and mean reinforcement. $J$. exp. Psychol., 1959, 57, 284-287.

\section{Note}

1. Support was provided by Grant HD 00906-05 from the National Institute of Health. 